\title{
¿Existe un Sesgo de Sobregeneralización y un Efecto de Positividad Relacionado con la Edad?
}

\author{
Is there an Overgeneralization Bias and an Age-Related Positivity Effect?
}

\author{
Juan C. Meléndez ${ }^{1}$, Ana I. Agusti ${ }^{2}$ y Encarnación Satorres ${ }^{3}$
}

\begin{abstract}
Resumen
Diversos estudios confirman cambios en el recuerdo de la memoria autobiográfica con el envejecimiento. Es interesante describir la manera en que el envejecimiento afecta la valencia y especificidad del recuerdo autobiográfico. Ochenta y siete adultos jóvenes y 75 adultos mayores fueron evaluados utilizando el AMT. Se realizaron dos ANOVA mixtos con dos grupos y tres tipos de memorias (específica, general, vaga) y tres valencias (positiva, negativa, neutra). El primero mostró efectos principales significativos para grupo, tipo de memoria e interacción, el segundo mostró efectos principales para grupo, valencia e interacción. Los jóvenes mostraron un mayor número de recuerdos específicos, y los adultos mayores produjeron más generales y vagos; para la valencia, los jóvenes obtuvieron mayor número de respuestas negativas, en comparación con el efecto de positividad de los adultos mayores. Se obtuvo un efecto de sobregeneralización en los adultos mayores, así como un efecto de positividad en la memoria autobiográfica.
\end{abstract}

Palabras clave: envejecimiento, memoria autobiográfica, efecto de positividad, especificidad de la memoria, valencia de la memoria

\begin{abstract}
Many studies support a change in the recall of autobiographical memories with aging. It is interesting to describe the way aging affects the valence and specificity of autobiographical recollection. Eighty-seven young adults and 75 older adults were assessed using the AMT. Two mixed ANOVA were performed with two groups and three types of memories (specific, general, vague), and three valences (positive, negative, neutral). The first showed significant main effects for group, type of memory, and interaction. The second showed main effects for group, valence, and interaction. Young people showed a greater number of specific memories, and older adults produced more general and vague responses; for valence, young people obtained a greater number of negative responses, as opposed to the positivity effect shown by the older adults. An effect of overgeneralization of memory was found in the older adults, as well as an effect of positivity on the autobiographical memory.
\end{abstract}

Keywords: aging, autobiographical memory, positivity effect, specificity of memory, valence of memory

\footnotetext{
${ }^{1}$ Doctor en Psicología. Profesor del Departamento de Psicología Evolutiva y de la Educación. Universidad de Valencia, España. Departamento de Psicología Evolutiva y de la Educación. Facultad de Psicología, Av. Blasco Ibáñez, 21, Valencia, España, 46010. Tel.: +34 963983844. Correo: melendez@uv.es

${ }^{2}$ Doctora en Psicología. Profesora del Departamento de Psicología. Universidad Internacional de Valencia, España. Pintor Sorolla, 21, Valencia, España, 46002. Correo: anaisabel.agusti@campusviu.es

${ }_{3}$ Doctora en Psicología. Profesora del Departamento de Psicología Evolutiva y de la Educación. Universidad de Valencia, España. Departamento de Psicología Evolutiva y de la Educación. Facultad de Psicología, Av. Blasco Ibáñez, 21, Valencia, España, 46010. Correo: encarna.satorres@uv.es
}

Revista Iberoamericana de Diagnóstico y Evaluación - e Avaliação Psicológica. RIDEP · No55 · Vol.2 · 151-160 2020

ISSN: 1135-3848 print /2183-6051online 


\section{Introducción}

El estudio de la cognición en el envejecimiento se ha desarrollado significativamente durante la última década, particularmente en relación con la memoria. En este contexto, el estudio de la memoria autobiográfica y los cambios que se producen con el envejecimiento es particularmente interesante dado que la memoria autobiográfica es parte de la memoria a largo plazo. La memoria autobiográfica representa un conjunto de informaciones y recuerdos sobre uno mismo que constituyen la historia personal y permiten construir un sentimiento de identidad y continuidad (Piolino, Desgranges, Benali, \& Eustache, 2002). La memoria autobiográfica es rica en pensamientos, emociones y evaluaciones sobre lo que sucedió, y proporciona marcos explicativos que contienen intenciones $y$ motivaciones humanas (Fivush, Habermas, Waters, \& Zaman, 2011).

De acuerdo con Tulving (2005) este tipo de memoria reúne conocimientos generales del pasado propio que constituyen un recuerdo objetivo en referencia al conocimiento del mundo que ha sido acumulado $y$ organizado conceptualmente (recuerdo semántico). Además, la memoria autobiográfica también incluye eventos específicos asociados con detalles perceptivos y sensoriales recopilados en el contexto de un tiempo y lugar específico, que se pueden afirmar de forma explícita, y que son activamente recuperados utilizando información contextual sobre cuándo y cómo se produjo (recuerdo episódico). Un factor determinante en la distinción entre estos dos tipos de recuerdo es que están asociados con estados de conciencia fundamentalmente diferentes. $\mathrm{La}$ memoria episódica se asocia con la conciencia autonoetica, que se considera una característica definitoria de la experiencia de recordar, un tipo de viaje mental en el tiempo donde se experimenta el pasado y el sentimiento de recordar se vuelve importante (Conway, 2005). La memoria semántica está asociada con la conciencia noética, e implica la toma de conciencia de hechos generales sobre eventos personales, acompañados de una sensación de simplemente "saber", sin detalles contextuales (Martinelli, Anssens, Sperduti, \&
Piolino, 2013); en los recuerdos semánticos, el conocimiento sobre el pasado se limita a la sensación de conocimiento o familiaridad.

Conway (2005) propuso un sistema conocido como Self-System Memory, el cual enfatiza la interconexión de uno mismo y la memoria para eventos autobiográficos. Este sistema contiene diferentes tipos de representaciones de memoria a largo plazo organizadas en una estructura jerárquica con tres niveles diferenciados: períodos de vida, eventos generales y conocimiento específico de eventos. Los períodos de vida son los diferentes espacios de tiempo que cada persona ha vivido, organizados en meses, años y décadas, y representan un conocimiento general sobre ese período. Los eventos generales habitualmente se organizan en días, semanas o meses, son más heterogéneos que los períodos de vida y se organizan en dos jerarquías: memorias extendidas, eventos que duran más de un día, y recuerdos categóricos, que se refieren a clases de eventos almacenados en categorías como personas, lugares o actividades. El nivel de conocimiento específico de eventos son recuerdos de episodios vinculados a un contexto espaciotemporal, es decir, registros esquematizados de los procesamientos sensorioperceptivos y conceptualafectivos propios de las experiencias concretas (Conway \& Pleydell-Pearce, 2000) y que contienen una gran cantidad de detalles. Conway (2005) propone que las estructuras de autoconocimiento de nivel superior, que abarcan largos períodos de tiempo, proporcionan acceso a información episódica sensorial-perceptiva de nivel inferior específica del evento.

Los recuerdos específicos parecen mostrar una disminución relacionada con la edad (Ferrante, 2004; Meléndez, Agusti, Satorres, \& Pitarque, 2018; Meléndez, Escudero, Satorres, \& Pitarque, 2019; Sánchez et al., 2019), mientras que la producción de detalles semánticos parece permanecer intacta o incluso mejorar en adultos mayores (Levine, Svoboda, Hay, Winocur, \& Moscovitch, 2002). Piolino et al. (2002) demostraron que la memoria autobiográfica episódica disminuye con la edad y la lejanía, mientras que se observa la persistencia de un almacén de memoria permanente de naturaleza semántica personal (es decir, información sobre eventos generales). Ros, Latorre y Serrano (2010) 
indican que los adultos mayores recuerdan un mayor número de recuerdos categóricos y un menor número de memorias específicas que los adultos jóvenes, lo que confirma la existencia de una sobregeneralización de la memoria, que ocurre en la población normal y aumenta con la edad. Sin embargo, otros estudios no muestran el efecto de la edad sobre la capacidad de recordar memorias episódicas remotas (Davidson \& Glisky, 2002).

Además, los recuerdos tienen una valencia asociada a ellos. Según la teoría de la selectividad socioemocional (TSS; Carstensen, 2006), asociado a la edad existe un efecto de positividad en el que se puede observar un aumento en la preferencia por la información positiva versus negativa en la atención y la memoria, en términos de diferencias relativas entre adultos jóvenes y adultos mayores (Mather \& Carstensen, 2005), donde los adultos mayores tienden a informar más recuerdos positivos que los adultos jóvenes (Gallo, Korthauer, McDonough, Teshale, \& Johnson, 2011). Sin embargo, la investigación sugiere que las entidades negativas tienen un mayor impacto psicológico que las positivas. Pratto y John (1991) indicaron que la información negativa puede necesitar más recursos de procesamiento que la información positiva y, por lo tanto, reduciría los recursos disponibles para procesar otros estímulos simultáneamente. Esta prioridad influiría en el sistema de memoria, de modo que la información negativa se recordaría con mayor precisión que la información positiva (Alves, Koch, \& Unkelbach, 2017), no evidenciándose el efecto de positividad. Kensinger, Garoff-Eaton y Schacter (2007) concluyeron que tanto los adultos mayores como los más jóvenes prestan más atención a los estímulos negativos que a los positivos, aunque las personas mayores lo hacen significativamente menos que las personas más jóvenes. Estas inconsistencias indican que el efecto de positividad en los adultos mayores puede no ser robusto.

El presente estudio examina la producción de recuerdos autobiográficos en adultos jóvenes y mayores, analizando el tipo de recuerdo y su valencia emocional. Se espera observar un mayor número de respuestas específicas que generales en los adultos jóvenes, mientras que, en los adultos mayores, debido a la sobregeneralización de la memoria, no debería haber diferencias entre ambos tipos de memoria. Además, al comparar los grupos, deberían existir diferencias a favor de los adultos jóvenes en las respuestas específicas, mientras que los adultos mayores deberían tener significativamente mayor número de recuerdos de tipo general. En el caso de las valencias, en el grupo de adultos jóvenes, se espera que obtengan una mayor producción de recuerdos con valencia negativa, mientras que, en los adultos mayores, se espera comprobar la hipótesis del efecto de positividad observado en otros estudios; finalmente estos efectos también deberían confirmarse al comparar los grupos.

\section{Método}

\section{Participantes}

Inicialmente la muestra estuvo compuesta por 165 participantes de Valencia (España), aunque al aplicar el criterio de exclusión de ingesta de medicamentos que afecten a la función cognitiva, se eliminaron 3 sujetos y la muestra fue finalmente de 162 participantes, diferenciándose dos grupos: 87 adultos jóvenes $(25.8 \%$ hombres, $74.2 \%$ mujeres), con edades comprendidas entre 18 y 27 años (media=20.64; $D T=2.54$ ), que eran estudiantes de psicología y magisterio de la Universidad de Valencia; y 75 adultos mayores (30.7\% hombres, $69.3 \%$ mujeres), con edades comprendidas entre 60 y 90 años (media=69.69; $D T=8.35$ ), que eran estudiantes de Nau Gran (extensión universitaria para personas mayores de 60 años) de la Universidad de Valencia.

En el grupo de adultos jóvenes, el $94.3 \%$ eran solteros y el $5.7 \%$ tenían otras situaciones de pareja; todos eran estudiantes universitarios. En el grupo de adultos mayores, $61.5 \%$ estaban casados, $7.7 \%$ eran solteros, $24.4 \%$ eran viudos y $6.4 \%$ estaban en otras situaciones de pareja; en términos de escolaridad, el $42.3 \%$ había terminado la escuela primaria, el $34.6 \%$ había terminado la escuela secundaria y el $23.1 \%$ había completado los estudios universitarios.

Antes de llevar a cabo la evaluación, todos los participantes en el estudio fueron informados sobre los objetivos y el procedimiento del estudio y dieron su consentimiento para participar de forma voluntaria. A continuación, se aplicó el Examen Cognoscitivo Mini-Mental (MEC; Lobo, 
Saz, \& Marcos, 2002) que es la versión en castellano del Mini-Mental State Examination (MMSE; Folstein, Folstein y McHugh, 1975). La puntuación en el MEC fue de 26 o superior en todos los casos, lo que indicó la inexistencia de signos observables de deterioro cognitivo. La media para los adultos mayores en el MEC fue 28.89 (rango 26-30) y, en comparación con el grupo de adultos jóvenes, no se observaron diferencias significativas. Además, los participantes completaron la Center for Epidemiologic StudiesDepression Scale (CES-D; Radloff, 1977); la puntuación obtenida en todos los participantes fue menor de 16, lo que indicó ausencia de sintomatología depresiva. Finalmente, se comprobó la ingesta de medicamentos que afectan la función cognitiva o antecedentes de abuso de alcohol o drogas evaluado mediante la Mini International Neuropsychiatric Interview (MINI, Sheehan et al., 1998). Se excluyeron tres adultos jóvenes con antecedentes de abuso de drogas.

\section{Materiales y procedimiento}

Todas las evaluaciones se realizaron individualmente. Primero, se recopilaron los datos sociodemográficos de los participantes, luego se verificó el cumplimiento de los criterios de exclusión y, finalmente, se aplicaron las pruebas. La sesión duró aproximadamente 1 hora. Los psicólogos a cargo de la evaluación fueron entrenados para administrar todas las pruebas usando el mismo procedimiento. Las evaluaciones de la memoria autobiográfica fueron registradas con una grabadora de voz para luego ser estudiadas por dos evaluadores independientes y poder verificar la confiabilidad entre jueces. Todas las pruebas utilizadas para la evaluación se enumeran a continuación.

El Examen Cognoscitivo Mini-Mental (MEC; Lobo et al., 2002) es una prueba de cribado que estima cuantitativamente la existencia y la gravedad del deterioro cognitivo sin proporcionar un diagnóstico de ninguna entidad nosológica específica. La puntuación máxima es de 30 puntos, obtenidos al sumar las puntuaciones de los diferentes ítems. El punto de corte para el deterioro cognitivo generalmente se establece en 23 puntos para las personas con escolaridad, ya que la prueba tiene una alta dependencia del lenguaje. Puntuaciones entre 23-21 indican demencia leve, entre 20-11 indican demencia moderada y las puntuaciones de 10 o menos indican demencia severa.

La Center for Epidemiologic StudiesDepression Scale (CES-D; Radloff, 1977) fue desarrollada como una medida de sintomatología depresiva en la población general. Esta escala tiene 20 elementos incluidos en escalas de depresión previamente validadas. En relación al formato de respuesta, para cada elemento, los participantes deben indicar el grado de acuerdo con la aparición del síntoma en una escala de frecuencia que varía de 0 (rara vez o nunca, menos de un día) a 3 (muchas veces o todo el tiempo, entre 5 y 7 días). Como punto de corte, generalmente se usa 16 , lo que indica la presencia de síntomas clínicamente significativos.

Mini International Neuropsychiatric Interview (MINI; Sheehan et al., 1998) es una entrevista diagnóstica estructurada a corto plazo que explora los principales trastornos psiquiátricos del Eje I del Manual Diagnóstico y Estadístico de los Trastornos Mentales (DSM-IV; APA, 1995) y la Clasificación Internacional de Enfermedades (CIE-10; OMS, 1993). El MINI consta de módulos para 17 diagnósticos psiquiátricos. Las preguntas están redactadas para permitir solo respuestas de "sí" o "no".

Autobiographical Memory Test (AMT; Williams y Broadbent, 1986). Esta prueba analiza la valencia y el tipo de recuerdo autobiográfico en respuesta a ciertas palabras clave. El procedimiento y las palabras clave utilizadas en este estudio se basaron en la adaptación de Ricarte, Latorre y Ros (2013) para muestras no clínicas. La lista definitiva consistía en cinco palabras con valencia positiva (felicidad, amistad, ilusión, energía y sonrisa), cinco con valencia negativa (culpable, fracaso, preocupación, tristeza, enfermedad, ) y dos palabras de tipo neutro que se ubican justo a la mitad de la prueba y al final de la misma (libro y llevar).Para su aplicación, a los sujetos se les presentaron las diferentes palabras clave, alternando valencias positivas y negativas (que además son contrabalenceadas), y se les pidió que intentaran evocar un recuerdo del pasado, lo más específico posible, asociado con cada una de las palabras. A continuación, se les explicó la diferencia entre un recuerdo específico y general, y se les proporcionó un ejemplo para 
ayudarlos a comprender esta diferencia. Las entrevistas se registraron con una grabadora de voz para su posterior codificación de acuerdo con dos categorías: valencia y especificidad. La valencia emocional, teniendo en cuenta la verbalización de la emoción que el sujeto expresó en ese momento, se clasificó como positiva, negativa o neutra (si el recuerdo evocado no expresaba ninguna emoción). Finalmente, la especificidad del recuerdo se clasificó como vaga cuando no hubo respuesta, general cuando no proporcionó información sobre el período específico (dónde y cuándo sucedió, etc.), o específica cuando el recuerdo se refirió a un día concreto, donde el participante describió lo que sucedió, lo que hizo y sintió, las circunstancias, con quién, dónde y cómo sucedió. Algunos autores, cuando analizan los recuerdos específicos, examinan el período de la vida al que se refiere la memoria, distinguiendo entre la infancia, la adolescencia, la juventud, la edad adulta y la vejez, y cuando no se refieren a ningún período específico; esta variable no se tuvo en cuenta en este estudio dada la imposibilidad de comparar grupos de adultos jóvenes y adultos mayores en algunos de estos períodos. Dos jueces independientes evaluaron individualmente las grabaciones para calcular la confiabilidad entre jueces. El total de las evaluaciones de los jueces se correlacionó con las 10 especificidades y 10 valencias de las palabras clave de AMT (usando la $r$ de Pearson). Las correlaciones de Pearson fueron $>.83$, señalando que la corrección es confiable.

\section{Análisis}

Se realizaron dos ANOVAS mixtos $(2 \times 3)$ con dos grupos (jóvenes y mayores sanos; intersujetos), y como factores intra-sujetos en primer lugar la especificidad del recuerdo (específico, general y vago) y en segundo lugar tres tipos de valencia (positiva, negativa y neutra), realizándose comparaciones post hoc con corrección de Bonferroni. Todos los análisis se realizaron con el programa SPSS 21.

\section{Resultados}

a. Especificidad de la memoria. Se realizó un primer ANOVA mixto con dos grupos (jóvenes y mayores; inter-sujetos) y tres tipos de recuerdo (especifico, general y vago; intra-sujetos). Los efectos principales del tipo de recuerdo $(F(2$, $\left.159)=31.45 ; \quad p<.001 ; \quad \eta^{2}=.283\right), \quad$ grupo $\quad(F(1$, $\left.160)=6.48 ; \quad p<.012 ; \quad \eta^{2}=.039\right), \quad$ así como la interacción $\left(F(2,159)=25.60 ; p<.001 ; \eta^{2}=.244\right)$, fueron significativos.

Debido a que la interacción fue significativa, se aplicaron pruebas de efectos simples (ver Tabla 1). El estudio de cada grupo analizado independientemente señaló un efecto significativo en el grupo de adultos jóvenes $(F(2,159)=60.67$; $\left.p<.001 ; \eta^{2}=.443\right)$ pero no en el grupo de adultos mayores $\left(F(2,159)=.82 ; p=.443 ; \eta^{2}=.010\right) . \mathrm{Al}$ aplicar pruebas post-hoc con corrección de Bonferroni en el grupo de jóvenes se obtuvo significativamente un mayor número de recuerdos específicos que generales $(p<.001)$ y que vagos $(p<.001)$, pero no se observaron diferencias significativas entre las respuestas generales y vagas.

Al comparar cada tipo de recuerdo entre los grupos se obtuvieron efectos simples significativos en: el recuerdo especifico $(F(1,160)=26.20$; $\left.p<.001 ; \eta^{2}=.141\right)$ donde los jóvenes produjeron un mayor número, y en el recuerdo general $(F(1$, $\left.160)=3.91 ; p=.049 ; \eta^{2}=.024\right)$ y en el vago $(F(1$, $\left.160)=57.34 ; p<.001 ; \eta^{2}=.264\right)$ donde los adultos mayores obtuvieron mayor cantidad de recuerdos.

Tabla 1. Media y desviación estándar de los grupos de acuerdo con el tipo de respuesta

\begin{tabular}{lccc}
\hline & Específica & General & Vaga \\
\hline $\begin{array}{l}\text { Adultos } \\
\text { jóvenes }\end{array}$ & $5.56(2.69)$ & $2.96(2.03)$ & $1.48(1.36)$ \\
$\begin{array}{l}\text { Adultos } \\
\text { mayores }\end{array}$ & $3.29(1.88)$ & $3.56(1.57)$ & $3.14(2.17)$ \\
\hline
\end{tabular}

b. Valencia de la memoria. Se realizó un segundo ANOVA mixto con dos grupos (jóvenes y mayores; intersujetos) por tres valencias (positiva, negativa y neutra), mostrando efectos principales significativos para la valencia $(F(2$, $\left.159)=1152.41 ; \quad p<.001 ; \quad \eta^{2}=.36\right)$, grupo $\quad(F(1$, $\left.160)=5.55 ; p<.020 ; \eta^{2}=.034\right)$, y para la interacción $\left(F(2,159)=53.83 ; p<.001 ; \eta^{2}=.405\right)$.

A continuación, y dada la interacción significativa, se aplicaron pruebas de efectos simples (ver Tabla 2). El estudio de cada grupo de forma independiente mostró un efecto significativo en el grupo de adultos jóvenes $(F(2$, 159)=572.68; $\left.p<.001 ; \eta^{2}=.879\right)$ así como en el 
grupo de adultos mayores $(F(2,159)=629.64$; $\left.p<.001 ; \eta^{2}=.889\right)$. Al aplicar pruebas post hoc con corrección de Bonferroni en el grupo de jóvenes se observó que producían significativamente mayor número de respuestas de tipo negativo que positivo $(p=.009)$ y neutro $(p<.001)$, y más respuestas positivas que neutras $(p<.001) . \mathrm{La}$ comparación post-hoc con corrección de Bonferroni en el caso de los adultos mayores mostro una mayor producción de respuestas positivas que negativas $(p<.001)$ y que neutras $(p<.001)$, y más respuestas negativas que neutras $(p<.001)$.

Al comparar cada una de las valencias en los grupos, se obtuvieron efectos simples significativos en la valencia negativa $(F(1,160)$ =28.97; $\left.p<.001 ; \eta^{2}=.154\right)$ donde los jóvenes produjeron mayor cantidad de respuestas, en la valencia positiva $(F(1,160)=77.05 ; \quad p<.001$; $\left.\eta^{2}=.326\right)$ donde los adultos mayores ofrecieron más respuestas, y no se observaron diferencias en las respuestas neutras $(F(1,160)=1.01 ; p=.323$; $\left.\eta^{2}=.006\right)$.

Tabla 2. Media y desviación estándar de los grupos según la valencia

\begin{tabular}{lccc}
\hline & Positiva & Negativa & Neutra \\
\hline $\begin{array}{l}\text { Adultos } \\
\text { jóvenes }\end{array}$ & $4.39(1.45)$ & $5.33(1.55)$ & $.28(.48)$ \\
$\begin{array}{l}\text { Adultos } \\
\text { mayores }\end{array}$ & $6.01(1.11)$ & $3.70(1.17)$ & $.29(.58)$ \\
\hline
\end{tabular}

\section{Discusión}

El objetivo principal de este estudio fue analizar el desempeño de adultos jóvenes y adultos mayores sanos en la prueba de AMT, a fin de estudiar la especificidad del recuerdo autobiográfico y su valencia. Como muestran los resultados, los jóvenes produjeron principalmente recuerdos específicos. Además, el número de recuerdos específicos fue significativamente mayor para el grupo de adultos jóvenes que para el de adultos mayores. En el grupo de adultos mayores, no hubo diferencias entre los diferentes tipos de recuerdos autobiográficos, aunque en comparación con los jóvenes, produjeron un número significativamente mayor de recuerdos generales y vagos. En cuanto a la valencia, los hallazgos verificaron que los jóvenes generaron principalmente respuestas negativas, mientras que los adultos mayores mostraron un sesgo positivo en sus recuerdos, observándose diferencias significativas en las valencias entre los dos grupos.

De acuerdo con Aizpurua y Koutstaal (2015) y Levine et al. (2002), la investigación sobre el envejecimiento cognitivo indica déficits relacionados con la edad en la información episódica, tales como detalles contextuales temporalmente específicos, mientras que la información semántica, como el conocimiento general, se conserva o incluso se facilita en los adultos mayores. En esta línea de resultados, fue posible verificar que los sujetos más jóvenes mostraron un claro componente de especificidad en sus recuerdos, mientras que en los adultos mayores no hubo diferencias entre los tipos de especificidad del recuerdo. Estos dos diferentes patrones de recuerdo pueden tener su base, de acuerdo con Ford, Rubin y Giovanello (2014), en la condición de la instrucción (recordar un acontecimiento específico o general); según estos autores, en los adultos mayores la instrucción no influiría en la recuperación, sugiriendo que no mantienen ni implementan las instrucciones de la tarea durante la recuperación; sin embargo, en los adultos jóvenes existiría una modulación en la recuperación que demostraría que utilizan la instrucción de la tarea alterando sus procesos de recuperación de la memoria para que coincidan con las instrucciones.

Al comparar los grupos, se observó una clara disminución del recuerdo específico en los adultos mayores, confirmando la hipótesis de la reducción de la memoria episódica asociada con el envejecimiento, mientras que los recuerdos generales y vagos se incrementaron significativamente en este grupo, permaneciendo independientes del efecto de edad. Según Piolino et al. (2002), el déficit de memoria episódica en las personas de más edad puede ser compensado con un aumento de los recuerdos semánticos (evento genérico). Como mencionaron estos autores y al igual que en este trabajo, el efecto es confiable porque el instrumento busca evocar, en la medida de lo posible, el recuerdo de memorias específicas y no genéricas. Para Piolino et al. (2006), los recuerdos de los adultos mayores, en comparación con los más jóvenes, suelen tratar 
eventos genéricos, repetidos y prolongados, sin ubicarlos en una situación espacio temporal o eventos que duran menos de un día y se recuperan sin detalles específicos. Como señalan Ros y Latorre (2010) y Ros et al. (2018), los procesos ejecutivos desempeñan un papel importante en la sobregeneralización de la memoria, y los cambios cognitivos inherentes a la vejez reducen la capacidad ejecutiva para recuperar recuerdos específicos, lo que explica por qué la sobregeneralización de la memoria es más evidente en adultos mayores que en adultos más jóvenes. Los recuerdos específicos se pueden recuperar mediante dos procesos: recuperación generativa y recuperación directa. La principal diferencia entre los dos tipos de recuperación es que el proceso de búsqueda está modulado por procesos de control en la recuperación generativa pero no, o no tan extensamente, en la recuperación directa. La recuperación generativa es un proceso de búsqueda de arriba hacia abajo en el que el sujeto utiliza representaciones conceptuales. La recuperación directa corresponde a la experiencia subjetiva del recuerdo espontáneo y se requieren menos recursos que la recuperación generativa. $\mathrm{La}$ sobregeneralización de la memoria surge cuando los individuos truncan su búsqueda durante la recuperación generativa a un nivel demasiado alto, accediendo solo a la información general. Williams (2006) desarrolló el modelo CaRFAX para explicar las causas de la aparición de la sobregeneralización de la memoria. Este modelo mostró que los déficits en las funciones ejecutivas limitan la recuperación exitosa de recuerdos específicos. La recuperación generativa requiere el uso de recursos ejecutivos para establecer un modelo de recuperación y compararlo con la información recuperada, e inhibir la información que es irrelevante para la memoria que se busca (Ros et al., 2018).

Con respecto a la valencia de la memoria, un primer resultado común para ambos grupos es la existencia de un mayor número de respuestas con contenido emocional que neutras. Este resultado es consistente con las propuestas de Adolphs, Tranel y Buchanan (2005), quienes informaron que la existencia de contenido emocional en la información aumenta la probabilidad de que se recuerde la esencia de la información.

Como muestra el presente estudio, los adultos jóvenes presentan principalmente recuerdos con una valencia negativa, siendo además significativamente superiores a los evocados por los adultos mayores. Kensinger et al. (2007) señalaron que los jóvenes muestran un beneficio general en el reconocimiento de elementos negativos (no positivos) en comparación con los neutros, señalando que el aumento de la memoria para los elementos negativos a menudo resulta en mejoras en la memoria o en el nivel de detalle. Por el contrario, cuando hay mejoras en la memoria para información positiva, es más probable que resulten de un mayor sentido de familiaridad o memoria de información general (no específica), generando a su vez un mayor número de falsas memorias (Pitarque, Sales, Meléndez, \& Algarabel, 2015).

En el grupo de adultos mayores, los resultados confirman la existencia de un efecto de positividad, con valores medios significativamente más altos para la valencia positiva que para la valencia negativa o neutra (Gallo et al., 2011; Reed \& Carstensen, 2012; Reed, Chan, \& Mikels, 2014), y mostrando un mayor número de recuerdos positivos que los jóvenes. La TSS postula que los adultos jóvenes, que perciben su tiempo como expansivo, se centran en objetivos relacionados con la precisión y la novedad para prepararse para futuros eventos. Por otro lado, los límites de tiempo percibidos por los adultos mayores conducen a un cambio en la motivación que lejos de buscar la precisión está más dirigido a metas emocionales (Mather \& Carstensen, 2005). Este cambio en los objetivos personales puede hacer que los adultos mayores se concentren en eventos positivos al recordar su pasado personal (Ford, DiGirolamo, \& Kensinger, 2016), y sugiere que los adultos mayores generalmente se centran en aspectos más positivos de las experiencias porque la generación de recuerdos autobiográficos en una tarea relativamente sin restricciones es sensible a las preferencias motivacionales. Según Mather y Carstensen (2005), los adultos mayores utilizan la reevaluación positiva como una estrategia para adaptarse a los eventos estresantes con mayor frecuencia que los adultos más jóvenes.

Aunque la mayoría de estos hallazgos empíricos se han interpretado desde la perspectiva de la TSS, Labouvie-Vief, Grühn y Studer (2010) proporcionaron una explicación alternativa $\mathrm{y}$ 
viable para este fenómeno empírico, basada en la Teoría de la Integración Dinámica. Según estos autores, con el envejecimiento y la pérdida del funcionamiento cognitivo fluido, la capacidad de relacionarse con información emocional altamente compleja se ve cada vez más comprometida. Por lo tanto, los adultos mayores prefieren el material positivo porque es más fácil de procesar (menos complejo) que la información negativa (Reed \& Cartensen, 2012).

Finalmente como limitaciones quiere señalarse el carácter transversal así como el tamaño de la muestra, que dificultan la generalización de las diferencias entre los grupos. Además, otra posible limitación, aunque de forma paralela una futura línea de trabajo, hace referencia al efecto de las instrucciones de las tareas en los resultados de sobregeneralización. Muchos estudios requieren que los participantes sean específicos en sus recuerdos. Según lo indicado por Ford et al. (2014), los adultos mayores tienen dificultades para seguir instrucciones específicas de memoria, mientras que esta modulación en la recuperación en adultos jóvenes señala que usan la instrucción durante la recuperación y alteran sus procesos de recuperación de memoria para que coincidan con las instrucciones. Por lo tanto, comparar los resultados de la memoria autobiográfica al establecer diferentes condiciones en las instrucciones podría ofrecer información relevante sobre cómo las instrucciones pueden modificar los resultados relacionados con la sobregeneralización de las respuestas en adultos mayores.

\section{Conflicto de interés}

Los autores no informan de ningún conflicto de intereses.

\section{Referencias}

Adolphs, R., Tranel, D., \& Buchanan, T. W. (2005). Amygdala damage impairs emotional memory for gist but not details of complex stimuli. Nature Neuroscience, 8, 512-518. doi:10.1038/nn1413

Aizpurua, A., \& Koutstaal, W. (2015). A matter of focus: Detailed memory in the intentional autobiographical recall of older and younger adults. Consciousness and Cognition, 33, 145155. doi:10.1016/j.concog.2014.12.006

Alves, H., Koch, A., \& Unkelbach, C. (2017). Why good is more alike than bad: Processing implications. Trends in Cognitive Sciences, 21, 69-79. doi:10.1016/j.tics.2016.12.006

American Psychiatric Association (1995). DSMIV. Manual diagnóstico y estadístico de los trastornos mentales. Barcelona: Masson.

Carstensen, L. L. (2006). The influence of a sense of time on human development. Science, 312, 1913-1915. doi:10.1126/science. 1127488

Conway, M. A. (2005). Memory and the self. Journal of Memory and Language, 53, 594628. doi:10.1016/j.jml.2005.08.005

Conway, M. A., \& Pleydell-Pearce, C. W. (2000). The construction of autobiographical memories in the self-memory system. Psychological Review, 107, 261-288. doi:10.1037/0033-295X.107.2.261

Davidson, P. S. R., \& Glisky, E. L. (2002). Is flashbulb memory a special instance of source memory? Evidence from older adults. Memory, 10, 99-111. doi:10.1080/09658210143000227

Ferrante, V. (2004). Indicadores cognitivos y perceptivos-motores de envejecimiento. Revista Iberoamericana de Diagnóstico y Evaluación - e Avaliação Psicológica, 18, 137-159.

Fivush, R., Habermas, T., Waters, T. E., \& Zaman, W. (2011). The making of autobiographical memory: Intersections of culture, narratives and identity. International Journal of Psychology, 46, 321-345. doi:10.1080/00207594.2011.596541

Folstein, M. F., Folstein, S. E., \& McHugh, P. R. (1975). Mini-mental state: A practical method for grading the cognitive state of patients for the clinician. Journal of Psychiatric Research, 12, 189-198. doi:10.1016/0022-3956(75)90026-6

Ford, J. H., DiGirolamo, M. A., \& Kensinger, E. A. (2016). Age influences the relation between subjective valence ratings and emotional word use during autobiographical memory retrieval. Memory, 24, 1023-1032. doi:10.1080/09658211.2015.1061016

Ford, J. H., Rubin, D. C., \& Giovanello, K. S. (2014). Effects of task instruction on autobiographical memory specificity in young 
and older adults. Memory, 22, 722-736. doi:10.1080/09658211.2013.820325

Gallo, D. A., Korthauer, L. E., McDonough, I. M., Teshale, S., \& Johnson, E. L. (2011). Agerelated positivity effects and autobiographical memory detail: Evidence from a past/future source memory task. Memory, 19, 641-652. doi:10.1080/09658211.2011.595723

Kensinger, E. A., Garoff-Eaton, R. J., \& Schacter, D. L. (2007). Effects of emotion on memory specificity in young and older adults. The Journals of Gerontology Series B: Psychological Sciences and Social Sciences, 62, 208-215.

Labouvie-Vief, G., Grühn, D., \& Studer, J. (2010). Dynamic integration of emotion and cognition: Equilibrium regulation in development and aging. In R. M. Lerner, M. E. Lamb, \& A. M. Freund (Eds.), The handbook of life-span development (Vol. 2, pp. 79-115). Hoboken, NJ: Wiley. doi:10.1002/9780470880166.hlsd002004

Levine, B., Svoboda, E., Hay, J. F., Winocur, G., \& Moscovitch, M. (2002). Aging and autobiographical memory: Dissociating episodic from semantic retrieval. Psychology and Aging, 17, 677-689. doi:10.1037/0882-7974.17.4.677

Lobo, A., Saz P., \& Marcos G. (2002). Adaptación del Examen Cognoscitivo MiniMental. Madrid, Spain: TEA Ediciones.

Martinelli, P., Anssens, A., Sperduti, M., \& Piolino, P. (2013). The influence of normal aging and Alzheimer's disease in autobiographical memory highly related to the self. Neuropsychology, 27, 69-78. doi:10.1037/a0030453

Mather, M., \& Carstensen, L. L. (2005). Aging and motivated cognition: The positivity effect in attention and memory. Trends in Cognitive Sciences, 9, 496-502. doi:10.1016/j.tics.2005.08.005

Meléndez, J. C., Agusti, A. I., Satorres, E., \& Pitarque, A. (2018). Are semantic and episodic autobiographical memories influenced by the life period remembered? Comparison of young and older adults. European Journal of Ageing, 15, 417-424. doi:10.1007/s10433-018-0457-4

Meléndez, J. C., Escudero, J., Satorres, E., \& Pitarque, A. (2019). Type of memory and emotional valence in healthy aging, mild cognitive impairment, and Alzheimer's disease. Psicothema, 31, 60-65. doi:10.7334/PSICOTHEMA2018.181

Organización Mundial de la Salud (1993). Clasificación estadística internacional de enfermedades y otros problemas de salud (CIE-10). Madrid: Meditor.

Piolino, P., Desgranges, B., Benali, K., \& Eustache, F. (2002). Episodic and semantic remote autobiographical memory in ageing. Memory, 10, 239-257. doi:10.1080/09658210143000353

Piolino, P., Desgranges, B., Clarys, D., GuilleryGirard, B., Taconnat, L., Isingrini, M., \& Eustache, F. (2006). Autobiographical memory, autonoetic consciousness, and selfperspective in aging. Psychology and Aging, 21, 510-525. doi:10.1037/0882-7974.21.3.510

Pitarque, A., Sales, A., Meléndez, J. C., \& Algarabel, S. (2015). Repetition increases false recollection in older people. Scandinavian Journal of Psychology, 56, 3844. doi:10.1111/sjop.12168

Pratto, F., \& John, O. P. (1991). Automatic vigilance: The attention grabbing power of negative social information. Journal of Personality and Social Psychology, 61, 380391. doi:10.1037/0022-3514.61.3.380

Radloff, L. S. (1977). The CES-D scale a selfreport depression scale for research in the general population. Applied Psychological Measurement, 1, 385-401. doi:10.1177/014662167700100306

Reed, A. E., \& Carstensen, L. L. (2012). The theory behind the age-related positivity effect. Frontier in Psychology, 3, 339. doi:10.3389/fpsyg.2012.00339

Reed, A. E., Chan, L., \& Mikels, J. A. (2014). Meta-analysis of the age-related positivity effect: Age differences in preferences for positive over negative information. Psychology and Aging, 29, 1-15. doi:10.1037/a0035194

Ricarte, J. J., Latorre, J. M., \& Ros, L. (2013). Design and performance analysis of Autobiographical Memory Test in Spanish population. [Diseño y análisis del funcionamiento del Test de Memoria 
Autobiográfica en población española]. Apuntes de Psicología, 31, 3-10.

Ros, L., \& Latorre, J. M. (2010). Gender and age differences in the recall of affective autobiographical memories using the autobiographical memory test. Personality and Individual Differences, 49(8), 950-954. doi:10.1016/j.paid.2010.08.002

Ros, L., Latorre, J. M., \& Serrano, J. P. (2009). Working memory capacity and overgeneral autobiographical memory in young and older adults. Aging, Neuropsychology, and Cognition, 17, 89-107. doi:10.1080/13825580903042650

Ros, L., Romero, D., Ricarte, J. J., Serrano, J. P., Nieto, M., \& Latorre, J. M. (2018). Measurement of overgeneral autobiographical memory: Psychometric properties of the autobiographical memory test in young and older populations. PloS one, 13(4), e0196073. doi:10.1371/journal.pone.0196073

Sánchez-Beato, A., Galindo, M., Gemeno, M., Jiménez, A., Prados Atienza, J. M., \& LópezHiges, R. (2019). Flexibilidad cognitiva y comprensión gramatical en mayores con deterioro cognitivo leve de tipo amnésico. Revista Iberoamericana de Diagnóstico y Evaluación - e Avaliação Psicológica, 50, 137-147. doi:10.21865/RIDEP50.1.11

Sheehan, D. V., Lecrubier, Y., Sheehan, K. H., Amorim, P., Janavs, J., Weiller, E., \& Lépine, J. P. (1998). The Mini-International Neuropsychiatric Interview (MINI): The development and validation of a structured diagnostic psychiatric interview for DSM-IV and ICD-10. Journal of Clinical Psychiatry, 59, 22-23.

Tulving, E. (2005). Episodic memory and autonoesis: Uniquely human. In S. Herbert \& J. Metcalfe (Eds.), The missing link in cognition: Origins of self-reflective consciousness (pp. 3-56). New York: Oxford University Press.

Williams, J. M. G. (2006). Capture and rumination, functional avoidance, and executive control (CaRFAX): Three processes that underlie overgeneral memory. Cognition and Emotion, 20(3-4), 548-568.

doi:10.1080/02699930500450465

Williams, J. M. G., \& Broadbent, K. (1986). Autobiographical memory in attempted suicide patients. Journal of Abnormal Psychology, 95, 144-149.

doi:10.1037/0021-843X.95.2.144 